\title{
Dynamic Changes of Traction Force at Focal Adhesions during Macroscopic Cell Stretching Using an Elastic Micropillar Substrate: Tensional Homeostasis of Aortic Smooth Muscle Cells*
}

\author{
Kazuaki NAGAYAMA**, Akifumi ADACHI** and Takeo MATSUMOTO** \\ ** Biomechanics Laboratory, Department of Mechanical Engineering, \\ Nagoya Institute of Technology, Gokiso-cho, Showa-ku, Nagoya 466-8555, Japan \\ E-mail: k-nagaym@nitech.ac.jp (K.N.), takeo@nitech.ac.jp (T.M.)
}

\begin{abstract}
In order to determine how cells change their traction forces at focal adhesions (FAs) under macroscopic deformation conditions, we investigated the dynamic changes in traction force at FAs by culturing porcine aortic smooth muscle cells (SMCs) on elastic micropillar substrates and giving them macroscopic deformation by stretching the substrates. We patterned adhesion region on the top surface of a polydimethylsiloxane-based micropillar array using our original micropatterning technique to align the cells on the pillar array parallel to the stretch direction. SMCs plated on the micropillars successfully spread in the adhesion region and their actin stress fibers (SFs) aligned in the direction to be stretched. Cells were then stretched and released cyclically with strain rates of $0.3 \% / 15 \mathrm{~s}$ up to $3-6 \%$ strain, and deflection of micropillars at both side regions of cells were measured simultaneously to obtain the traction force at each FA in situ. SMCs aligned in the stretch direction showed two types of responses: almost a half of the SMCs changed their force in phase with the applied strain, and showed gradual active contraction with the stretch cycles (synchronous group); and the rest tended to keep their force constant and became elongated with the cycles (asynchronous group). In the asynchronous group, the force sometimes changed in antiphase with the cell strain as if the cells maintain intracellular traction force at a constant level. These results may indicate that SMCs sometimes exhibit active homeostatic responses to keep their pretension constant during macroscopic stretching, and such tensional homeostatic responses may occur concurrently with cell elongation.
\end{abstract}

Key words: Cell Biomechanics, Mechanical Properties, Stress Fibers, Force Transmission, Cytoskeleton

\section{Introduction}

Adherent cells generate traction forces through contraction of the actin cytoskeleton, and transmit this force via focal adhesions (FAs) to the underlying extracellular matrix (ECM). Cellular traction forces contribute to the mechanical properties ${ }^{(1)}$ and shape stability ${ }^{(2)}$ of cells, and they are believed to play pivotal roles in regulating various cellular events such as cell proliferation ${ }^{(3)}$, migration ${ }^{(4,5)}$, differentiation ${ }^{(6)}$, and remodeling of the $\mathrm{ECM}^{(7)}$.

Existence of the cellular traction force was first demonstrated by plating cells onto a thin deformable substrate of a polydimethylsiloxane (PDMS) sheet. Forces generated by cells were visualized as wrinkles in the sheet ${ }^{(8,9)}$. The traction force was also estimated in cells

[DOI: 10.1299/jbse.7.130]

Copyright (C) 2012 by JSME 
on soft substrates by embedding fluorescent beads within the substrate and tracking the bead displacement ${ }^{(10)}$. This technique demonstrated that the magnitude and direction of traction forces correlated with the size and direction of FAs to which the forces were applied. Tan et al. ${ }^{(11)}$ established another traction force measurement technique utilizing a PDMS-based elastic substrate with an array of micropillars, in which cells were cultured on the pillar array and the traction force was detected by the deflection of the pillars. The advantage of this technique is that each micropillar acts as an individual cantilever sensor attached to FAs. However, these studies focused only on the cellular traction forces in a static culture condition. Adherent cells such as vascular smooth muscle cells (SMCs) are dynamically exposed to macroscopic cyclic stretching in the vascular wall in vivo. For a detailed quantification of mechanical signal transduction mechanisms, it is crucial to determine dynamic changes in traction force at each FA during macroscopic deformation of cells.

For this purpose, we recently developed a technique to measure the dynamic changes in traction forces at each FA during macroscopic stretch of the cells. We cultured SMCs on the micropillar array and stretched the cells by stretching the substrate under a microscope stage, and revealed that the force at FAs of SMCs showed a heterogeneous response during macroscopic cell stretching ${ }^{(12)}$. However, the direction of the cell alignment was not controlled in the previous study. The mechanical response of cells may depend on the orientation of the cells because cells have mechanical anisotropy depending on the alignment of actin stress fibers (SFs) ${ }^{(13,14)}$. Thus, evaluation of the mechanical responses of cells considering cell orientation is quite important.

In the present study, we established an experimental technique to measure dynamic changes in traction forces at FAs of cells during macroscopic stretching with adjustment of cell orientation. For this purpose, we developed a micropatterning technique to align the direction of the cell on the pillars parallel to the stretch direction, and adapted it to our cell stretching protocol. We then cultured porcine aortic SMCs on the elastic micropillar array substrate, performed a loading/unloading test repeatedly for SMCs aligned in the stretch direction, and investigated the mechanical response of SMCs.

\section{Materials and Methods}

\subsection{Preparation of cultured smooth muscle cells}

Porcine aortic SMCs were obtained by an explant method previously described ${ }^{(15)}$, and were cultured in Dulbecco's Modified Eagle's Medium (DMEM, Invitrogen, USA) supplemented with $10 \%$ fetal bovine serum (JRH Bioscience, USA), penicillin (100 unit $/ \mathrm{ml}$ ), and streptomycin $(100 \mu \mathrm{g} / \mathrm{ml})\left(\right.$ Sigma) at $37^{\circ} \mathrm{C}$ in $5 \% \mathrm{CO}_{2}$ and $95 \%$ air. The cells were passaged repeatedly at a $1: 4$ split ratio when they reached $\sim 80 \%$ confluence. Cells at passage 5-9 were used for all experiments.

\subsection{Fabrication of the cell culture chamber with micropillars}

Micropillar array substrates were fabricated by replica-molding (11) as previously described ${ }^{(16)}$. Briefly, an array of photoresist posts was made on a silicon wafer by standard photolithography as a male mold. To make a female mold for the array of micropillars, polydimethylsiloxane (PDMS; Sylgard 184, Dow-Corning) was poured over the male mold, cured at $70^{\circ} \mathrm{C}$ for $6 \mathrm{~h}$, peeled off, and silanized to aid release of PDMS from the female mold. PDMS was then poured into the mold, cured at $100^{\circ} \mathrm{C}$ for $3 \mathrm{~h}$, and peeled off to obtain the micropillar array substrate. Young's modulus of the substrate was measured by a laboratory-built elastometer based on the pipette aspiration test ${ }^{(17)}$, and was $1.6 \pm 0.1 \mathrm{MPa}$ (mean $\pm \mathrm{SD}, \mathrm{n}=27$ ). We used a hexagonal arrangement micropillar array whose pillar diameter, length, and center-to-center spacing were 3, 9, and $9 \mu \mathrm{m}$, respectively (Fig. 1A).

The PDMS micropillar array substrate $(23 \times 23 \times 1 \mathrm{~mm}$ with a pillar region of $10 \times 15$ $\mathrm{mm}$ ) was glued to the bottom of a rectangular silicone rubber frame $(23 \times 44 \times 10 \mathrm{~mm}$ and 
$20 \times 20 \times 10 \mathrm{~mm}$ as outside and inside dimensions, respectively) (Fig. 1C). The substrate was sterilized with UV light for $1-2 \mathrm{~h}$ in a clean bench. In a separate study, we confirmed that the UV irradiation did not affect the substrate stiffness. In order to confirm the morphology of SMCs cultured on the pillar substrate, we coated fibronectin $(50 \mu \mathrm{g} / \mathrm{ml}$, Sigma) onto the top surface of the pillars using the microcontact printing method ${ }^{(18)}$, and treated the side wall of pillars with $2 \%$ bovine serum albumin (BSA) for $1 \mathrm{~h}$ to prevent cell adhesion. SMCs were then cultured on the substrates in the DMEM (10\% FBS) for 3-8 $\mathrm{h}$ until they spread fully. We confirmed that most $(>80 \%)$ SMCs were located on the top surface of the pillars and their spreading produced deflection of micropillars, showing distribution of tractions forces at FAs (Fig. 1B). Traction forces $F$ were determined using the simple equation $F=k \delta$, where $k$ and $\delta$ are the spring constant and deflection of the micropillars, respectively. The spring constant of the pillars $k$ was obtained from the average Young's modulus of the substrate $(1.6 \mathrm{MPa})$ and the pillar geometry, and was 26 $\mathrm{nN} / \mu \mathrm{m}$ in the present study.
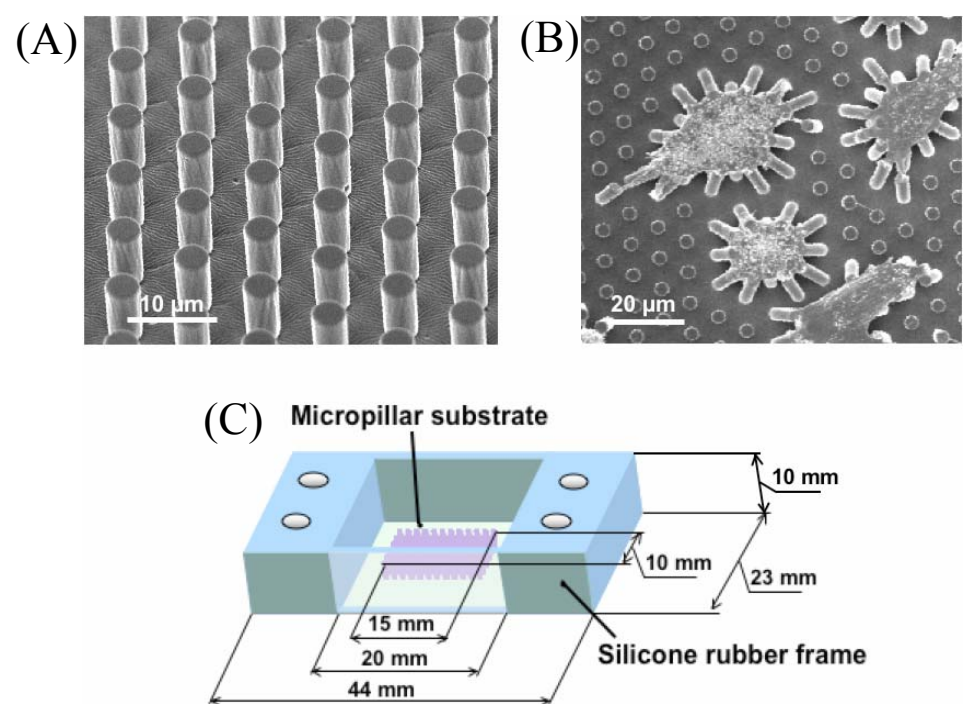

Figure 1: Scanning electron micrograph of a substrate with a micropillar array (A) and SMCs spread on top of the micropillars (B). Cell culture chamber with a micropillar substrate for macroscopic stretching $(\mathrm{C})$.

\subsection{Alignment of cells on pillars using a micropatterning technique}

In order to align SMCs to the stretch direction, we adjusted the adhesion region of the micropillar array substrate using the following method (Fig. 2A): we attached a metal specimen grid for transmission electron microscopy (TEM grid, Ni 100/400, VECO) with rectangular openings $(220 \mu \mathrm{m} \times 30 \mu \mathrm{m})$ firmly to the surface of a PDMS sheet $(t=\sim 2 \mathrm{~mm})$. We then poured a fibronectin solution $(50 \mu \mathrm{g} / \mathrm{ml}$, Sigma $)$ onto the PDMS sheet through the holes of the grid, air-dried them in a clean bench for 3-6 h, and then peeled off the grid from the PDMS sheet. We then adjusted the long axis direction of the fibronectin-coated area of the PDMS sheet to the stretch direction, and attached the coated surface onto the top of the pillars for $\sim 10 \mathrm{~min}$ to make the rectangular adhesion regions (Fig. 2B). After a blocking process with 2\% BSA described in Section 2.2, we plated SMCs sparsely onto the pillars in the DMEM with $10 \%$ FBS for $\sim 6 \mathrm{~h}$. SMCs elongated within the rectangular area and aligned in the stretch direction (Fig. 2C). However, multiple cells spread onto the same patterned area (Fig. 2C, arrowheads) in some cases, because the rectangle opening of the TEM grid used in this study $\left(6600 \mu \mathrm{m}^{2}\right)$ was larger than the area of SMCs spreading on the pillars $\left(2525 \pm 600 \mu \mathrm{m}^{2}\right.$, mean $\left.\pm \mathrm{SD}, \mathrm{n}=80\right)$. We omitted such cells and used SMCs spreading alone in each rectangular area for mechanical tests. 

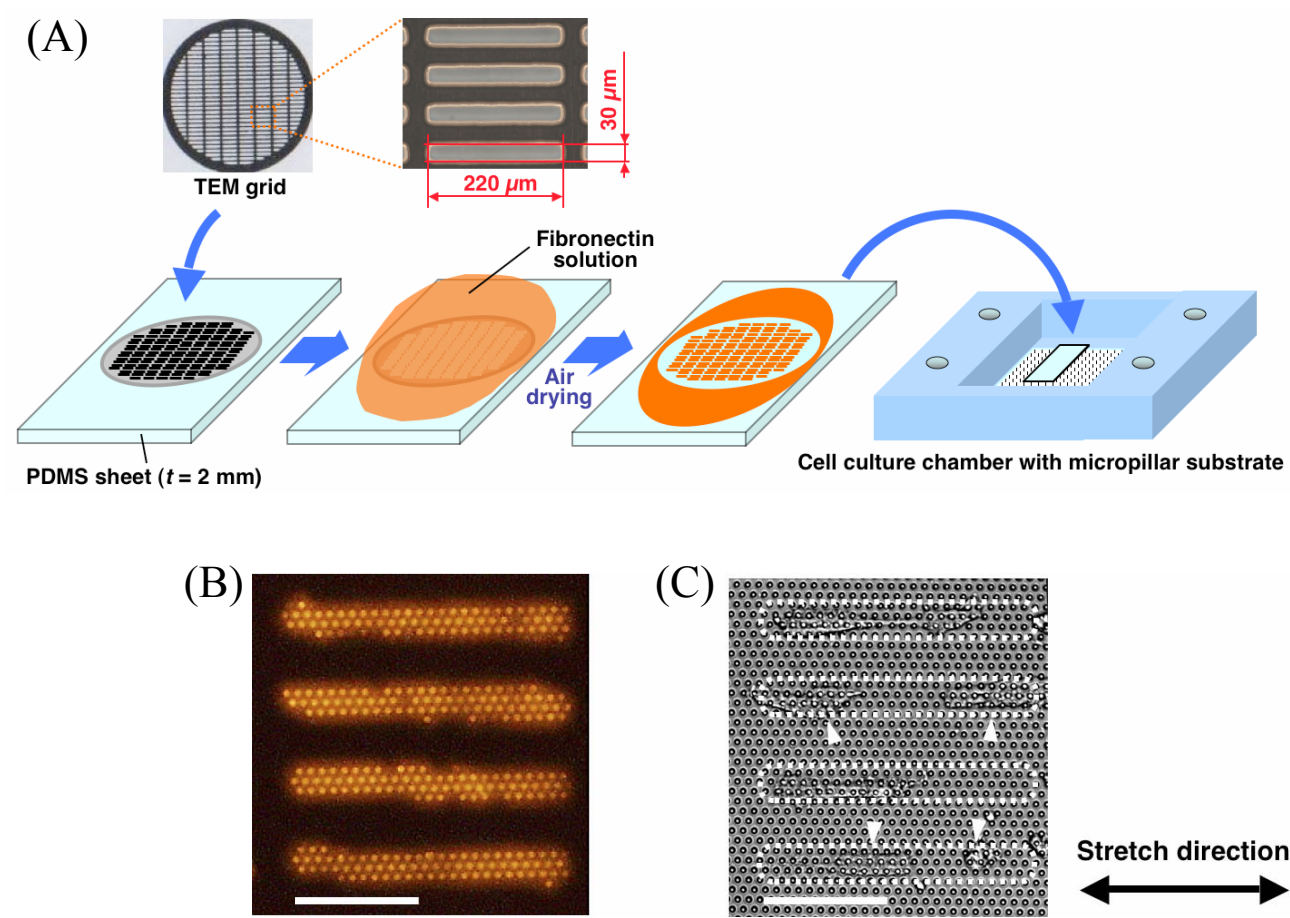

Stretch direction

Figure 2: The micropatterning process to make the rectangular adhesion region of a micropillar array substrate (A). A typical image of micropillars whose top ends have been coated with fibronectin (B) and SMCs adhering onto these pillars (C). Note that the micropatterned areas of fibronectin were visualized fluorescently with Alexa fluor 546 in (B), and SMCs elongated along these micropatterned areas $(C)$. Dotted rectangular areas in $(C)$ represent the micropatterned areas. Bars $=100 \mu \mathrm{m}$.

\subsection{Cell stretching apparatus}

A cell stretching apparatus (STB-150, Strex, Japan) set on the stage of an inverted microscope (TE2000E, Nikon, Japan) was used. In its original configuration, the cell culture chamber is fixed on one side and stretched to another side by a moving holder driven by a stepping motor, and cells in the chamber move out of the field of view easily when stretched. To overcome this problem, we modified the apparatus so as to stretch cells symmetrically using a mechanism with wires and pulleys (Fig. 3). We could observe the same cell continuously in the chamber during stretching under a 40x objective ${ }^{(12)}$. The temperature around the chamber was kept at $37^{\circ} \mathrm{C}$ by heated water in the bath. The $\mathrm{pH}$ of the culture medium was maintained at 7.4 with a gas mixture of $5 \% \mathrm{CO}_{2}$ and $95 \%$ air.

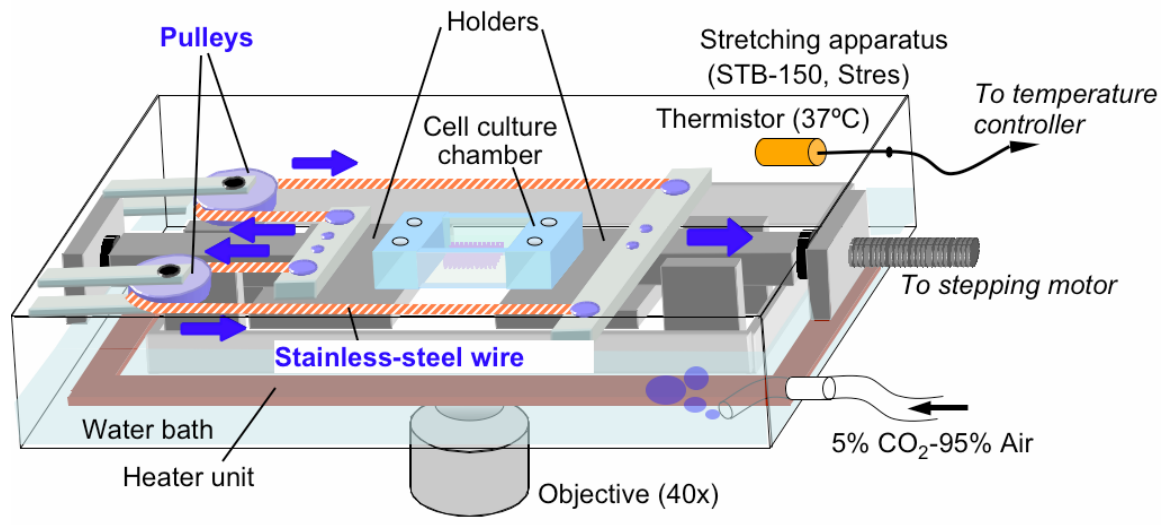

Figure 3: Schematic representation of the main part of the apparatus used to stretch the cell culture chamber. A symmetric stretching mechanism consisting of wires and pulleys was added to the original stretching apparatus. 


\subsection{In-situ measurement of the traction force of SMCs during macroscopic stretching}

SMCs on the pillars in the culture chambers were set on the stretching apparatus and pre-incubated for $1 \mathrm{~h}$ at $37^{\circ} \mathrm{C}$. Cells were then stretched by stretching the substrate incrementally by $0.3 \%$ every $15 \mathrm{~s}$ and then unloaded in a similar manner. The cells and their neighboring pillars were observed with a cooled digital CCD camera (ORCA-ER, Hamamatsu Photonics, Japan) connected to the microscope during stretch and release, and time-course images of SMCs and pillars were recorded on a personal computer. Deflection of pillars was measured on the recorded images with multiparticle tracking software (G-track, G-angstrom, Japan). In bright field images, the brightness of the pillar was highest at the center of the pillar tip and gradually decreased to the periphery as represented by a Gaussian curve (Fig. 4A). Thus, two-dimensional Gaussian fitting ${ }^{(19)}$ was used to determine the position of the center in the software. Displacement of the pillar tip from its unloaded position was used as its deflection to calculate traction forces $(F)$ at FAs. We recently established a method to estimate unloaded position of the pillars during stretching without removing the cells from the pillars ${ }^{(12)}$ : we estimated the $\mathrm{X}-\mathrm{Y}$ coordinates of the unloaded position of the target pillars deflected by the cells using the X-Y coordinates of adjacent pillars that did not attach to the cells (Fig. 4B). We also measured the strain of the substrate in the stretch direction as the whole-cell strain $\varepsilon_{\text {cell }}$. In this study, we stretched cells from $\varepsilon_{\text {cell }}$ $=0$ to $3 \%$ three times (Cycles 1-3) and then from $\varepsilon_{\text {cell }}=0$ to $6 \%$ three times (Cycles 4-6) to investigate the effect of strain magnitude on the mechanical response of the SMCs. Traction forces of an SMC were generated mainly towards the center of the cell. The traction force was especially high at the cell periphery $(20-80 \mathrm{nN})$, and very low under the nucleus $(<$ $10 \mathrm{nN})^{(12,16)}$. We thus focused on the traction forces at both side regions of SMCs (Fig. 4C, the region of interest (ROI) was set at the parallelogram containing 4 rows and 3 columns of the pillars), measured their time-course changes during stretch/release cycles, and avaraged the traction force at pillars attaching to the cells in ROI as an index of the response at the whole-cell level.

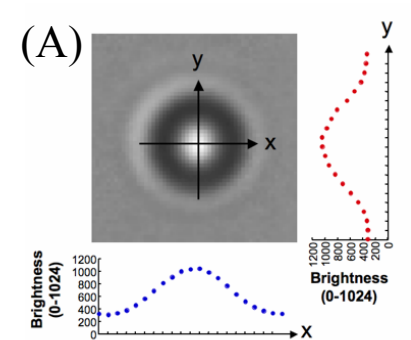

(C)
(B)

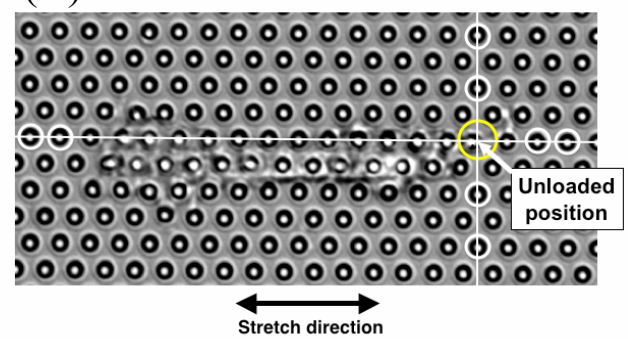

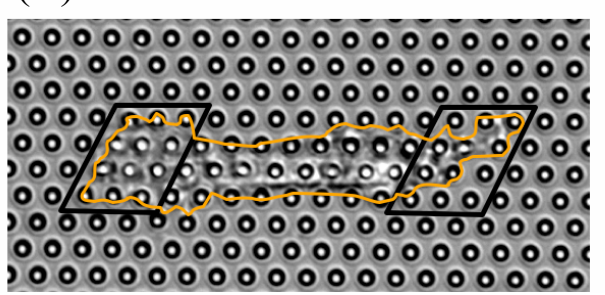

Figure 4: An example of a bright field image of a micropillar and its distribution of brightness in the $\mathrm{x}$ and $\mathrm{y}$ directions (A). The distribution profile can be fitted well with Gaussian distribution curves. An example of an image of an SMC on the micropillar substrate, and estimation of the $\mathrm{x}-\mathrm{y}$ coordinates of the unloaded position of a target pillar bent by cell traction force (B, yellow circle). We measured the $x-y$ coordinates of unloaded pillars ( $B$, white circles) whose row and column were the same as those of the target pillar in all image stacks, and estimated the $x-y$ coordinates of the unloaded position of the target pillar. In this study, the region of interest (ROI) for time-course measurement of traction forces at FAs was set at both side regions of the cells (C, parallelograms). Dark yellow line in $(\mathrm{C})$ represents the outline of the cell. 


\subsection{Statistical analysis}

Data are expressed as mean \pm SD. Differences were analyzed by the Student's paired and unpaired $t$-test and were considered significant when $\mathrm{P}<0.05$.

\section{Results}

Figure 5 shows typical images of an SMC elongated along the micropatterned area of the pillar substrate (A) and fluorescent images of its actin filaments (B). During stretch/release cycles repeated 6 times, the micropatterned SMCs maintained their morphology and did not detach markedly from the pillar surface. SMCs spreading on the pillars showed clearly organized thick SFs anchoring at the top of pillars, and the fibers aligned in the stretch direction (B). Changes in the traction forces at some pillars during the stretch/release cycles are shown in Fig. 5C. The changes in the forces of the micropatterned SMCs during stretch were almost similar to those of unpatterned SMCs reported in our previous study ${ }^{(12)}$, and changes were quite heterogeneous among locations: the force changed in phase with the applied strain $\varepsilon_{\text {cell }}$ at some pillars (L2, white arrows, Cycles 4-6), in antiphase at other pillars (R1 and 2, black arrows, Cycles 4-6), and was almost independent from the strain at the other pillars (L1).

(A)

\section{L1}

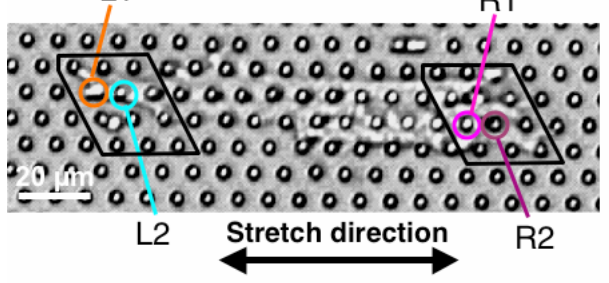

(B)

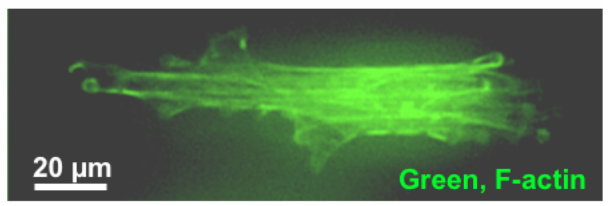

(C)
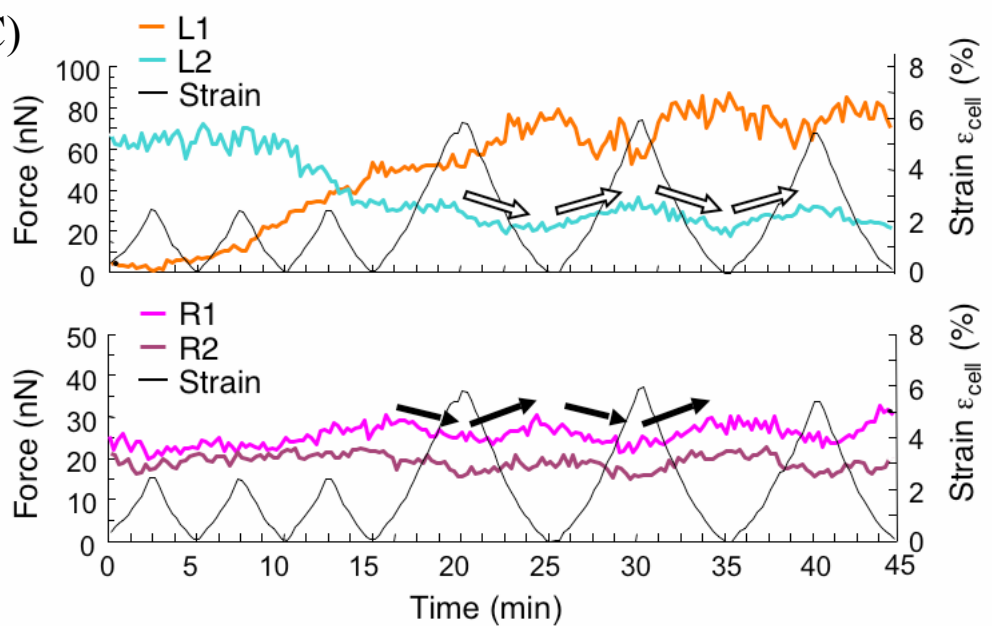

Figure 5: An example of an SMC on the elastic micropillar substrate just after stretch and release (A), and fluorescent images of its actin filaments (B). To confirm the distribution of actin stress fibers, we stained some cells with Alexa Fluor-conjugated phalloidin (Molecular Probes) just after the stretch experiments. Time-course changes of the whole-cell strain $\varepsilon_{\text {cell }}$ and the traction force $F$ at FAs (C) during stretch/release of the SMC shown in A. 
The change in the average value of traction force at both side regions of SMCs varied from cell to cell (Fig. 6A), and was roughly classified into two types of responses: synchronous and asynchronous. In the synchronous group, the average force increased and decreased in synchronization with the applied strain $\varepsilon_{\text {cell }}$ (Cell 1-4), and in the asynchronous group, the force was almost independent from $\varepsilon_{\text {cell }}$ (Cell 5-8). The peak force in each cycle was larger in Cycles 4-6 than in Cycles 1-3 for the synchronous group, while in the asynchronous group the force tended to be constant among all cycles. The force in the asynchronous group looked smaller than that of the synchronous group, and it was slightly in anti-phase with the strain in some cases (Fig. 6A, arrows). SMCs looked contracted and elongated following stretch/release cycles in the synchronous and asynchronous groups, respectively (Fig. 6B and $\mathrm{C}$, upper and middle panels). However, no significant difference was observed in the morphology of SF in the both groups (Fig. 6B and C, lower panels).

(A)

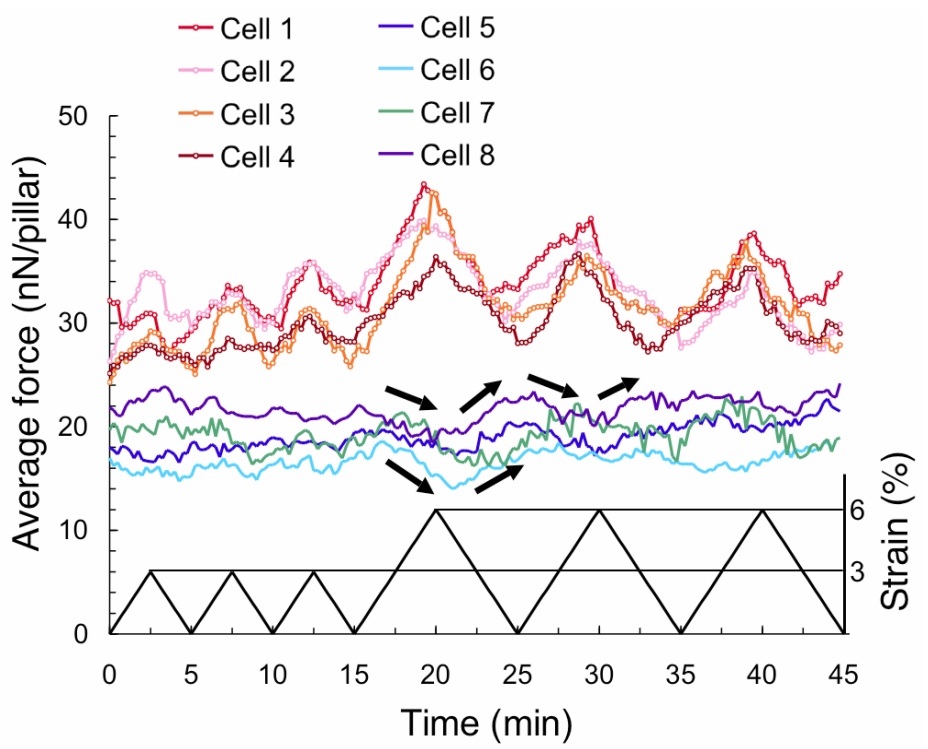

(B)

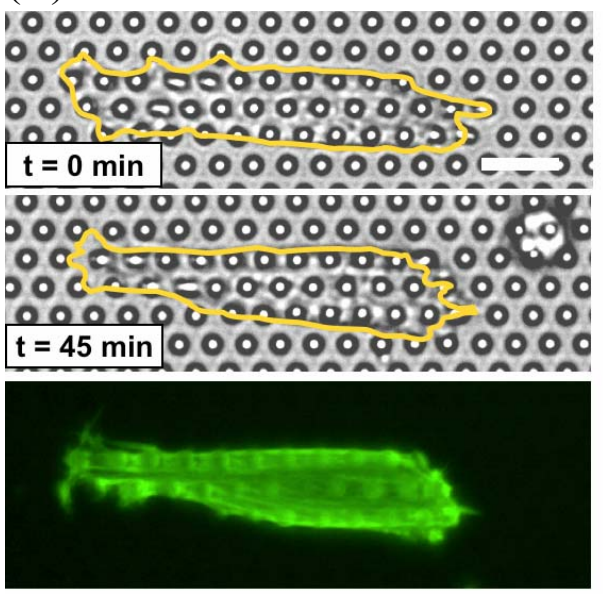

Cell 1
(C)

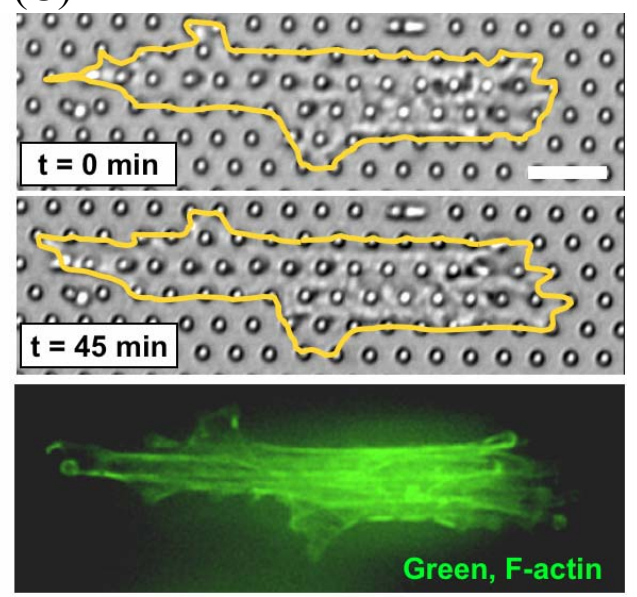

Cell 5

Figure 6: Time-course changes in the average value of traction force at FAs of both side regions of SMCs (A) during stretch and release cycles, and typical images of SMCs in the synchronous and asynchronous groups ( $\mathrm{B}$ and $\mathrm{C}$, respectively) before (upper) and just after (middle) the stretch and release tests, and their fluorescent images of actin filaments (lower). Bars = $20 \mu \mathrm{m}$. Note that the strain shown in A represents the preset value (not the actually measured value as in Fig. 5C). Dark yellow lines in B and C represent the outlines of cells. 
Figure 7 shows changes in the average value of traction force at $\varepsilon_{\text {cell }}=0$, which represents the cell pretension applied to each pillar at both side regions of SMCs, and in the cell area at $\varepsilon_{\text {cell }}=0$ in both groups. The pretension of synchronous SMCs was $\sim 50 \%$ larger than that of asynchronous SMCs (A). The cell area showed opposite changes between the two groups: it tended to decrease and increase with the number of stretch/release cycles in the synchronous and asynchronous groups, respectively (B), and a statistically significant difference was observed in the relative area for the larger strain cycles (C, Cycles 4-6).
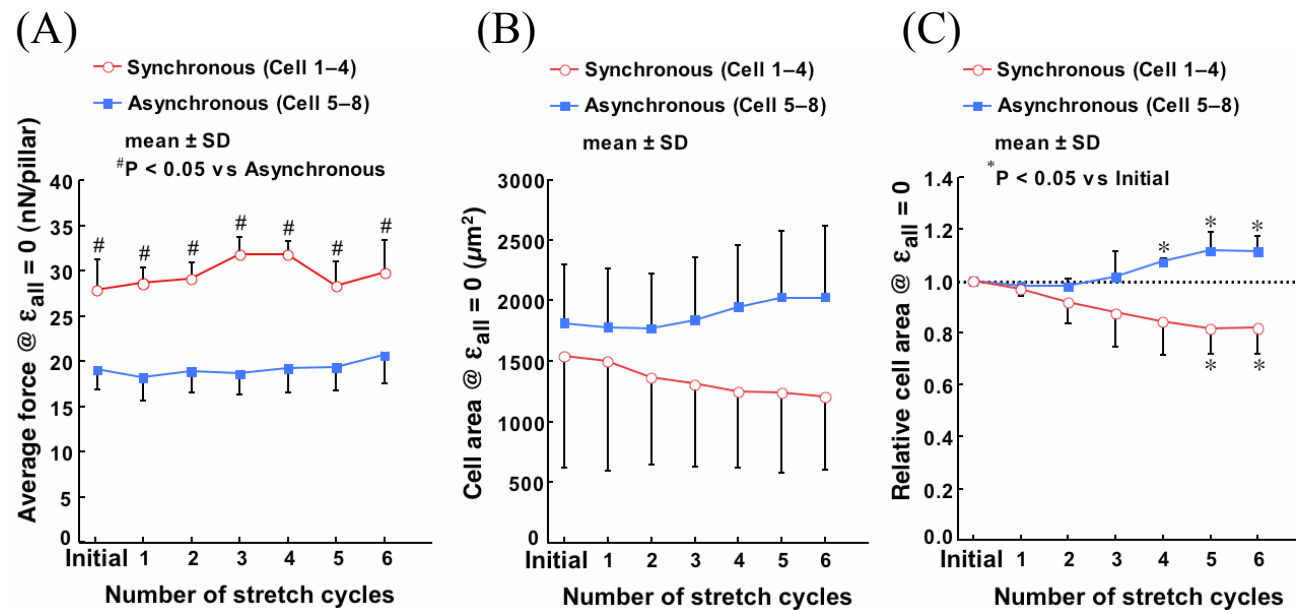

Figure 7: Changes in the average value of traction force at FAs of both side regions of SMCs at $\varepsilon_{\text {cell }}=0$ (pretension at FAs) during the stretch and release cycles (A), and in cell area at $\varepsilon_{\text {cell }}=$ 0 (B and $\mathrm{C}$ ) for both groups.

\section{Discussion}

We successfully established a technique to measure the dynamic changes of traction force at individual FAs of a cell during macroscopic stretching in its longitudinal axis using an elastic micropillar substrate micropatterned to control cell orientation. We previously measured changes in traction forces at FAs of SMCs on the micropillar substrate by stretching the substrate, and revealed that the force at FAs of SMCs showed a heterogeneous response during stretching ${ }^{(12)}$. However, in the previous study, we did not use the micropatterning technique and chose cells whose major axis direction was within $30^{\circ}$ of the stretching direction. Since SMCs exhibit mechanical anisotropy depending on the alignment of actin stress fibers (SFs) ${ }^{(13,14)}$, the mechanical properties of SMCs, which affect the mechanical response of cells, may be dependent on the orientation of the cells. To overcome this problem, we controlled the adhesion region of the top surface of pillars using a TEM grid as a mask to make the micropatterns of fibronectin (Fig. 2B). Although the rectangle holes of the TEM grid $\left(\sim 6500 \mu \mathrm{m}^{2}\right)$ were larger than the average area of SMCs spreading on the pillars $\left(\sim 2500 \mu \mathrm{m}^{2}\right)$, we achieved a good alignment of SMCs and their SFs to the stretch directions (Fig. 5B). Artery walls are stretched cyclically by $\sim 10 \%$ to their circumferential direction due to the pulse pressure. Thus, SMCs that align almost parallel to the circumferential direction of the walls are also exposed to such stretch conditions. Our experimental technique is therefore quite useful in mimicking such a mechanical environment of SMCs in aortic walls and allowing the investigation of their mechanical response in vivo.

In our previous study, the mechanical response of unpatterned SMCs on the micropillars showed a heterogeneous response of forces at FAs during stretch/release cycles, which 
might have been caused by the diversity of orientation of their SFs anchoring to the pillars ${ }^{(12)}$. This heterogeneous response was also observed in micropatterned SMCs in the present study, even though their SFs were aligned in the stretch direction (Fig. 5C). Such aligned SFs in micropatterned SMCs looked attached to several pillars in the same lines. It has recently been suggested that ventral SFs were attached to the substrate at various adhesion sites ${ }^{(20)}$, and that SFs may have shown heterogeneous contraction due to the heterogeneous distribution of non-muscle myosin and $\alpha$-actinin ${ }^{(21)}$. These structural differences observed in single SFs may result in a heterogeneous response of the traction force at each FA during macroscopic stretching.

In this study, we calculated the average value of traction force at both side regions of SMCs to assess the mechanical response at the whole-cell level. The force changed in phase with the applied strain in some cells (synchronous group), while they were almost independent in the other cells (asynchronous group). In the asynchronous group, the forces tended to be constant during the stretch/release cycles, and they sometimes slightly in antiphase with the cell strain (Fig. 6A, arrows). It has been reported that surface stiffness of fibroblasts measured using an atomic force microscope significantly increased (or decreased) following a single $8 \%$ step uniaxial stretch (or compression), and that it returned gradually toward baseline values ${ }^{(22)}$. Na et al. ${ }^{(23)}$ later investigated changes in the surface stiffness of SMCs adhering onto the flat surface of a PDMS sheet, and demonstrated that SMCs transiently increased their stiffness when they were exposed to equibiaxial cyclic stretching with $10 \%$ strain at $0.25 \mathrm{~Hz}$, and were restored within $5 \mathrm{~min}$. These previous studies may indicate that cells show active homeostatic responses during macroscopic deformation to maintain their constant stiffness, which is regulated by cell pretension. The force responses of the asynchronous SMCs observed in this study may also represent such mechanical homeostatic responses.

A larger pretension was observed in the synchronous SMCs, and the cell area tended to decrease with the number of stretch/release cycles. In contrast, pretension was lower in the asynchronous SMCs, and the cell area increased with the number of stretch/release cycles (Fig. 7). The detailed mechanisms of these opposite phenomena are unclear at this stage. It has been pointed out that the Rho GTPase family is activated by mechanical stretching, and that it regulates actin dynamics which are implicated in cell contraction and elongation ${ }^{(24)}$. The activated level of Rho GTPase plays a role in determining cell contraction and elongation ${ }^{(25)}$. These findings indicate that the activation level of Rho GTPase may also affect the elongation and contraction of SMCs during the stretching/release cycles and that it switches the mechanical responses of SMCs. In an effort to increase our understanding of this phenomenon, we are now planning to investigate the relation between changes in traction force at FAs, intracellular actin dynamics, and the Rho-dependent pathway. Mechanical response of cells also might be influenced by the cell cycle, because the morphology of SFs and cell adhesion forces change during cell cycle ${ }^{(26)}$. Thus we also need to consider the effects of cell cycle on the mechanical response of SMCs in future studies.

\section{Conclusions}

We established a novel experimental technique to measure dynamic changes in traction forces at FAs of cells during macroscopic stretching and release in their longitudinal direction, and investigated the mechanical response of SMCs. We found that SMCs aligned in the stretch direction showed two types of responses: the traction force changed in phase with the applied strain in some cells (synchronous group), while they were almost independent in the other cells (asynchronous group). We also found that SMCs sometimes exhibit active homeostatic responses to keep their pretension constant during macroscopic stretching, and such tensional homeostatic responses may occur concurrently with cell 
elongation. To make clear the mechanism of these responses, we need to investigate the change in traction force at FAs, the dynamics of SFs and the Rho-dependent pathway in future studies.

\section{Acknowledgements}

This work was supported in part by MEXT and JSPS KAKENHIs (nos. 20680025 and 22650104 to K.N., and nos. 22127008 and 22240055 to T.M.), Japan.

\section{References}

[1] Suresh, S., Biomechanics and biophysics of cancer cells, Acta Biomaterialia, Vol. 3, No. 4 (2007). pp. 413-438.

[2] Satcher, R., Dewey, Jr. C.F., and Hartwig, J.H., Mechanical remodeling of the endothelial surface and actin cytoskeleton induced by fluid flow. Microcirculation, Vol.4 (1997), pp. 439-453.

[3] Chen, C.S., Mrksich, M., Huang, S., Whitesides, G.M., and Ingber, D.E., Geometric control of cell life and death, Science, Vol. 276-5317 (1997), pp. 1425-1428.

[4] Galbraith, C.G., and Sheetz, M.P., A micromachined device provides a new bend on fibroblast traction forces, Proceedings of the National Academy of Sciences USA, Vol. 94, No. 17 (1997), pp. 9114-9118.

[5] Burton, K., Park, J.H., and Taylor, D.L., Keratocytes generate traction forces in two phases, Molecular Biology of the Cell, Vol. 10, No. 11 (1999), pp. 3745-3769.

[6] Li, B., Lin, M., Tang, Y., Wang, B., and Wang, J.H., A novel functional assessment of the differentiation of micropatterned muscle cells, Journal of Biomechanics, Vol. 41, No. 16 (2008), pp. 3349-3353.

[7] Rabinovitz, I., Gipson, I.K., and Mercurio, A.M., Traction forces mediated by alpha6beta4 integrin: implications for basement membrane organization and tumor invasion, Molecular Biology of the Cell, Vol. 12, No. 12 (2001), pp. 4030-4043.

[8] Harris, A.K., Wild, P., and Stopak, D., Silicone rubber substrata: a new wrinkle in the study of cell locomotion, Science. Vol. 208, (1980), pp. 177-179.

[9] Harris, A.K., Jr. Tissue culture cells on deformable substrata: biomechanical implications. Journal of Biomechanical Engineering, Vol. 106 (1984), pp. 19-24.

[10] Dembo, M., and Wang, Y.L., Stresses at the cell-to-substrate interface during locomotion of fibroblasts, Biophysical Journal, Vol. 76 (1999), pp. 2307-2316.

[11] Tan, J.L., Tien, J., Pirone, D.M., Gray, D.S., Bhadriraju, K., and Chen C.S., Cells lying on a bed of microneedles: an approach to isolate mechanical force, Proceedings of the National Academy of Sciences USA, Vol. 100, No. 4 (2003), pp. 1484-1489.

[12] Nagayama, K., Adachi, A., and Matsumoto, T., Heterogeneous response of traction force at focal adhesions of vascular smooth muscle cells subjected to macroscopic stretch on a micropillar substrate, Journal of Biomechanics, Vol. 44, No. 15 (2011), pp. 2699-2705.

[13] Nagayama, K., and Matsumoto, T., Mechanical anisotropy of rat aortic smooth muscle cells decreases with their contraction: possible effect of actin filament orientation, JSME International Journal, Series C, Vol. 47 (2004), pp. 985-991.

[14] Hu, S., Eberhard, L., Chen, J., Love, J.C., Butler, J.P., Fredberg, J.J., Whitesides, G.M., and Wang, N., Mechanical anisotropy of adherent cells probed by a three-dimensional magnetic twisting device, American Journal of Physiology, Cell Physiology, Vol. 287, No.5 (2004), C1184-1191.

[15] Nagayama, K., Tsugawa, A., and Matsumoto, T., Tensile properties of cultured aortic 
smooth muscle cells obtained in a quasi-in situ tensile test with thermoresponsive gelatin, Journal of Biomechanical Science and Engineering, Vol. 1 (2006), pp. 256-267.

[16] Nagayama, K., and Matsumoto, T., Dynamic change in morphology and traction forces at focal adhesions in cultured vascular smooth muscle cells during contraction, Cellular and Molecular Bioengineering Vol. 4, No. 3 (2011), pp. 348-357.

[17] Aoki, T., Ohashi, T., Matsumoto, T., and Sato, M., The pipette aspiration applied to the local stiffness measurement of soft tissues, Annals of Biomedical Engineering, Vol. 25, No. 3 (1997), pp. 581-587.

[18] Chen, C.S., Mrksich, M., Huang, S., Whitesides, G.M., and Ingber, D.E., Micropatterned surfaces for control of cell shape, position, and function, Biotechnology Progress, Vol. 14, No. 3 (1998), pp. 356-363.

[19] du Roure, O., Saez, A., Buguin, A., Austin, R.H., Chavrier, P., Silberzan, P., and Ladoux, B., Force mapping in epithelial cell migration, Proceedings of the National Academy of Sciences USA, Vol. 102, No. 7 (2005), 2390-2395.

[20] Colombelli, J., Besser, A., Kress, H., Reynaud, E.G., Girard, P., Caussinus, E., Haselmann, U., Small, J.V., Schwarz, U.S., and Stelzer, E.H., Mechanosensing in actin stress fibers revealed by a close correlation between force and protein localization, Journal of Cell Science, Vol. 122(Pt 10) (2009), 1665-1679.

[21] Peterson, L.J., Rajfur, Z., Maddox, A.S,, Freel, C.D,, Chen, Y., Edlund, M., Otey, C., and Burridge, K., Simultaneous stretching and contraction of stress fibers in vivo, Molecular Biology of the Cell, Vol. 15, No.7 (2004), pp. 3497-3508.

[22] Mizutani, T., Haga, H., and Kawabata K., Cellular stiffness response to external deformation: tensional homeostasis in a single fibroblast, Cell Motility and Cytoskeleton, Vol. 59 (2004), pp. 242-248.

[23] Na, S., Trache, A., Trzeciakowski, J., Sun, Z., Meininger, G.A., and Humphrey, J.D., Time-dependent changes in smooth muscle cell stiffness and focal adhesion area in response to cyclic equibiaxial stretch, Annals of Biomedical Engineering, Vol. 36, No. 3 (2008), pp. 369-380.

[24] Riveline, D., Zamir, E., Balaban, N.Q., Schwarz, U.S., Ishizaki, T., Narumiya, S., Kam, Z., Geiger, B., and Bershadsky, A.D., Focal contacts as mechanosensors: externally applied local mechanical force induces growth of focal contacts by an mDial-dependent and ROCK-independent mechanism, Journal of Cell Biology, Vol. 153, No. 6 (2001), pp. 1175-1186.

[25] Arakawa, Y., Bito, H., Furuyashiki, T., Tsuji, T., Takemoto-Kimura, S., Kimura, K., Nozaki, K., Hashimoto, N., and Narumiya, S., Control of axon elongation via an SDF-1alpha/Rho/mDia pathway in cultured cerebellar granule neurons, Journal of Cell Biology, Vol. 161, No. 2 (2003), pp. 381-391.

[26] Weder, G., Vörös, J., Giazzon, M., Matthey, N., Heinzelmann, H., and Liley, M., Measuring cell adhesion forces during the cell cycle by force spectroscopy, Biointerphases, Vol. 4, No. 2 (2009), pp. 27-34. 